\title{
In Silico Characterization and Homology Modeling of a Cyanobacterial Phosphoenolpyruvate Carboxykinase Enzyme
}

\author{
Aubrey A. Smith and Amanda Caruso \\ Department of Biology, Montgomery College-Rockville Campus, 51 Mannakee Street, Rockville, MD 20850, USA \\ Correspondence should be addressed to Aubrey A. Smith; aubrey.smith@montgomerycollege.edu
}

Received 19 October 2012; Revised 18 January 2013; Accepted 4 February 2013

Academic Editor: Manuela Helmer-Citterich

Copyright ( 2013 A. A. Smith and A. Caruso. This is an open access article distributed under the Creative Commons Attribution License, which permits unrestricted use, distribution, and reproduction in any medium, provided the original work is properly cited.

ATP-dependent phosphoenolpyruvate carboxykinase (PEPCK) is a key catabolic enzyme found in various species of bacteria, plants, and yeast. PEPCK may play a role in carbon fixation in aquatic ecosystems consisting of photosynthetic cyanobacteria. RuBisCO-based $\mathrm{CO}_{2}$ fixation is prevalent in cyanobacteria through $\mathrm{C}_{3}$ intermediates; however, a significant amount of carbon flows into $\mathrm{C}_{4}$ acids during cyanobacterial photosynthesis. This indicates that a $\mathrm{C}_{4}$ mechanism for inorganic carbon fixation is prevalent in cyanobacteria with PEPCK as an important $\beta$-carboxylation enzyme. Newly available genomic information has confirmed the existence of putative PEPCK genes in a number of cyanobacterial species. This project represents the first structural and physicochemical study of cyanobacterial PEPCKs. Biocomputational analyses of cyanobacterial PEPCKs were performed and a homology model of Cyanothece sp. PCC 7424 PEPCK was generated. The modeled enzyme consists of an N-terminal and C-terminal domains with a mixed $\alpha / \beta$ topology with the active site located in a deep cleft between the two domains. Active site residues and those involved in metal ion coordination were found to be conserved in the cyanobacterial enzymes. An active site lid which is known to close upon substrate binding was also predicted. Amino acid stretches that are unique to cyanobacterial PEPCKs were also identified.

\section{Introduction}

Phosphoenolpyruvate carboxykinase (PEPCK; EC 4.1.32) catalyzes the reversible ATP- or GTP-dependent decarboxylation of oxaloacetate (OAA) to yield phosphoenolpyruvate (PEP). This reaction uses the phosphate group from the nucleotide triphosphate and, as a result, produces $\mathrm{CO}_{2}$ and the corresponding nucleoside diphosphate. PEPCK has a strict requirement for divalent cations with $\mathrm{Mn}^{2+}$ as its best activator [1]. Two classes of PEPCKs exist in nature, and they are classified in the basis of the nucleotide substrate: ATPutilizing enzymes are found in bacteria, plants, and yeast, while GTP-dependent PEPCKs are found mostly in higher eukaryotes [2]. GTP-dependent PEPCKs also occur in some bacteria such as Corynebacterium glutamicum [3]. While there is no significant sequence identity between the two classes, a number of residues are completely conserved across all PEPCKs in the regions of the enzyme that are necessary for nucleotide binding and metal ion coordination [1]. The crystal structures of PEPCKs from representative species of plants, bacteria, and mammals have been published, and conservation in metal and substrate binding were confirmed [4].

In mammals, two forms of PEPCKs exist: PEPCK-M, which is expressed in mitochondria, and PEPCK-C, the cytosolic form of the enzyme. Mammalian PEPCK plays a major role in gluconeogenesis and glyceroneogenesis. The enzyme is also a contributor to other downstream processes [7]. The significance of the PEPCK reaction has been studied in Streptococcus bovis, Selenomonas ruminantium, and other bacteria where PEPCK was found to be involved in growth initiation and amino acid synthesis [8]. E. coli overexpressing PEPCK exhibited slower growth rate and increased ATP production [9]. PEPCK has also been investigated as the sole anaplerotic enzyme in the yeast Saccharomyces cerevisiae [10]. In plants, where PEPCK isoforms are expressed in various tissues, regulation of the enzyme by phosphorylation is dependent upon location and illumination [11]. The same is true for CAM plants such as Ananas comosus [12]. 
Cya8801 Cyaccy0110 Cya7424 Thermus A. succi

E. coli

A. thal 1

A. thal2

Z. mays

T. cruzi

H. sapiens

Rattus

Gallus

C. glut
16 LKIAPHLSQGLDYPKVHPSSVTT SDREDGIKFPTYGLETLGIHNVSWVYRNLPVAELVEH QPNLSLSSQ----PSYSGNT TLEKDSEEAIIRPT YGLEDLGLYNI GRVYRNLPIAQLVEH 16 TLPGQSKTNHLAGCSEAITPPNYLVEND TFHQPT YGLESLGMKNLGRVFHNLSVPQLVEQ

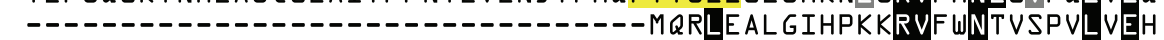

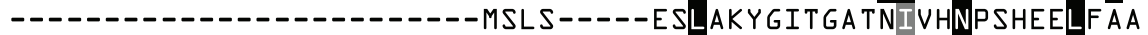
. KVVRGDPAEK--KT DGSTTPAYAHGQHHSIFSPATGAVSDSSLKF THVLYNLSPAELYEQ 13 KLIRGDP--------TSAAKVAHVPVTPTSLPAADVSD SGLKF THILHNLSPAELYEQ 100 KVVKGDPAAKGEAAAQGAPSTPRAHQQHRHPAAPAIAVSD SSLKF THVLNNLSPAELYEQ 1 43 IHICDGSEEENGRLLGQMEEEGILRRLK--KYDNCWLALT DPRDVARIESKTVIVTQEQR 1 1 - - GKELLRGLQD D GVLHPLP--KY DNCWLART DPR DVARVQSKTVLVTPEQS 36 VVFVDGSQAEWDRMAEDLVEAGTLIKLNEEKRPNSYLARSNPSDVARVESRTFICSEKEE

\begin{tabular}{|c|c|c|}
\hline Сya8801 & 121 & HHNVAISERQFEQLYQRVLSYVQGR-ELY IFDG--YVGADRNYRHGVRVINELASQNL \\
\hline Cyaccy0110 & 117 & LHNVPISEAKFNQ YQKVIA QGR-DIYIFDG--YIGADTNYRCGVRIVNELASQNL \\
\hline Сya7424 & 121 & DNVPISEAKFEQ \\
\hline Thermus & 73 & $\mathrm{HA}$ \\
\hline A. succi & 85 & \\
\hline E. coli & 92 & \\
\hline A. thall & 212 & NIEMDE \\
\hline A. thal 2 & 211 & NIEMDE \\
\hline Z. mays & 208 & NWDPENRIKVRIIS \\
\hline T. cruzi & 65 & GHDERYRLKVRVF \\
\hline H. sapiens & 155 & $G(P$ \\
\hline Rattus & 85 & KIGIELTDS \\
\hline Gallus & 104 & KLGVQVT DSPYVVL \\
\hline C. glut & 142 & \\
\hline
\end{tabular}

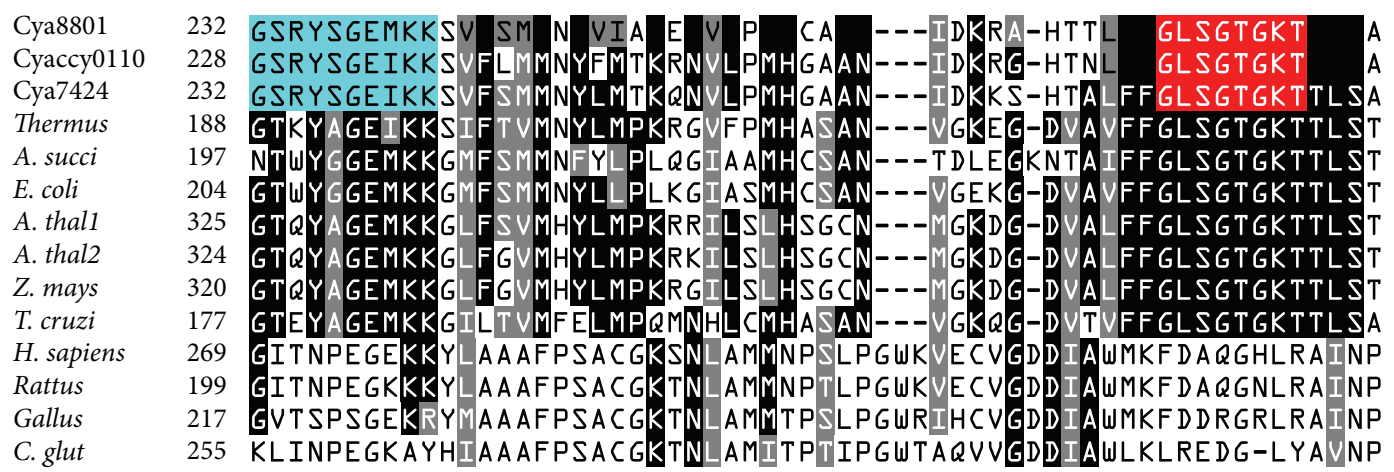

Figure 1: Continued. 


\begin{tabular}{|c|c|c|}
\hline Суа8801 & 288 & DPDCSLIGD DEHGWSKDGIFNFEGG. \\
\hline Cyaccy0110 & 284 & DPDCHLIG D DEHGWSESGIFNFEGG--CYAKTI \\
\hline Cya7424 & 288 & -IGDDEHGWSDDGVFNFEG--CYAKTI \\
\hline Thermus & 244 & $\bar{S} P \bar{E} H \overline{E P} L \overline{I Y Y} K \overline{A S} N Q \overline{F E} A \overline{\text { ILENVVVN }}$ \\
\hline A. succi & 254 & DPKRLLIGDDEHGWDDDGVFNFEGG--C \\
\hline E. coli & 260 & DPKRRLIGDDEHGWDDDGVNFEGG- - \\
\hline A. thall & 381 & 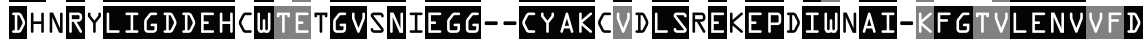 \\
\hline A. thal2 & 380 & $\bar{D} H N \bar{R} Y \overline{L I G D D E H} C \overline{W S E A} \overline{G V} S \bar{N} I \overline{E G G}$ \\
\hline Z. mays & 376 & 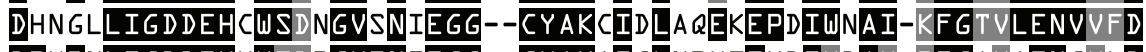 \\
\hline T. cruzi & 233 & $\bar{V} D \overline{I Y} D \overline{A V}-\overline{R F} A \overline{A E N C V L \bar{D}}$ \\
\hline H. sapiens & 329 & -NTIFTNVAETSDGGVYWEGIDEPLASGVT ITSWK \\
\hline Rattus & 259 & NT IF TNVAETSDGGVYWEG I DEPLAPGVT I T SWK \\
\hline Gallus & 277 & PTEPGVTYTSW \\
\hline C. glut & 314 & G--DAPAHLIDWM \\
\hline
\end{tabular}

\begin{tabular}{|c|c|c|}
\hline y8801 & 463 & TQVFLLNT GWSGGSYGVG \\
\hline Сyaccy0110 & 459 & PIF V \\
\hline Cya7424 & 463 & VFLINT GWSGGRYGVG- \\
\hline Thermus & 420 & TRALLKAALSGALENVPYRRDPVFGFEV \\
\hline A. succi & 429 & TRGI IDAILDGSIDTANT A T IP \\
\hline E. coli & 435 & DTRAIIDAI \\
\hline A. thal1 & 556 & T-GWLVNT GWSGGSYGVG. \\
\hline A. thal2 & 555 & SGGSYGTG \\
\hline Z. mays & 551 & T-GWLVNTGWSG \\
\hline T. cruzi & 409 & R-VULLNT GY AGGRA DRGAKRMPLRVTRAIIDAI \\
\hline H. sapiens & 507 & GKASTKLTP \\
\hline Rattus & 437 & LWPGF GENSRVLEWMFGRI \\
\hline Gallus & 455 & EF \\
\hline C. glut & 492 & \\
\hline
\end{tabular}

FIGURE 1: Multiple sequence alignment of selected regions of ATP- and GTP-dependent PEPCKs. The yellow block highlights are amino acid stretches that are conserved and exclusive to cyanobacterial PEPCKs; the amino acids in the blue blocks make up the pyruvate binding site; the kinase-la and kinase 2 motifs are highlighted by the red and pink boxes, respectively; the active site lid amino acids are shaded in green; the adenine binding site is highlighted in olive green. The alignment was generated with Clustal W [5].

PEPCK is also prevalent in marine ecosystems where a significant amount of $\mathrm{CO}_{2}$ fixation takes place. The enzyme is involved in light-dependent carbon fixation, and its activity varies with changes in water temperature and nitrogen concentration [13]. Furthermore, PEPCK contributes to photosynthetic carbon fixation as an addition to the $\mathrm{C}_{3}$ pathway catalyzed by RuBisCO [14]. The same may be said of marine cyanobacteria where $\beta$-carboxylation reactions involving phosphoenolpyruvate carboxylase (PEPC) and PEPCK are prevalent [15].

Cyanobacteria are unable to derive metabolic energy from the Krebs cycle since they lack $\alpha$-ketoglutarate dehydrogenase and NADH oxidase. Furthermore, $\mathrm{C}_{4}$ acid concentration fluctuates in cyanobacteria in response to lightdark cycles. This indicates that $\mathrm{C}_{4}$ mechanisms for inorganic carbon fixation involving PEPC and PEPCK are widespread 
TABLE 1: Phosphoenolpyruvate carboxylase sequences retrieved from the NCBI database (http://www.ncbi.nlm.nih.gov/).

\begin{tabular}{lcc}
\hline Organism & $\begin{array}{c}\text { Accession } \\
\text { number }\end{array}$ & $\begin{array}{c}\text { Nucleotide } \\
\text { specificity }\end{array}$ \\
\hline Cyanothece sp. PCC 8802 & ACV02875.1 & ATP \\
Cyanothece sp. PCC 7424 & ACK71186.1 & ATP \\
Cyanothece sp. PCC 8801 & ACK67979.1 & ATP \\
Cyanothece sp.ATCC 51142 & ACB49859.1 & ATP \\
Microcystis aeruginosa NIES-843 & BAG00438.1 & ATP \\
Cyanothece sp. CCY0110 & EAZ91985.1 & ATP \\
Cyanothece sp. PCC 7822 & ADN17293.1 & ATP \\
Microcoleus chthonoplastes PCC & EDX72609.1 & ATP \\
7420 & CAO87080 & ATP \\
Microcystis aeruginosa PCC 7806 & ZP-06380836 & ATP \\
Arthrospira platensis str. Paraca & AAA50780.1 & ATP \\
Trypanosoma cruzi & BAE77888.1 & ATP \\
E. coli K12 & AAC45394.1 & ATP \\
A. succiniciproducens & ABB53264.1 & GTP \\
C. glutamicum & AAH23978.1 & GTP \\
Homo sapiens & AAH81900.1 & GTP \\
Rattus norvegicus & NP-990802 & GTP \\
Gallus gallus & YP-143544.1 & ATP \\
Thermus thermophilus HB8 & &
\end{tabular}

in those organisms [16]. In marine ecosystems, PEPCK may be involved in light-independent carbon fixation (LICF) in diatoms [17].

Despite their potential importance in net $\mathrm{CO}_{2}$ fixation, PEPC and PEPCK from cyanobacteria have not been extensively studied. A model of PEPC from a marine cyanobacterium is available, but no such model exists for cyanobacterial PEPCK [18]. In this work, a homology model for a cyanobacterial PEPCK is presented for the first time. Furthermore, the enzymes' physicochemical properties were characterized in silico.

\section{Materials and Methodology}

2.1. PEPCK Protein sequences. Phosphoenolpyruvate carboxykinase (PEPCK) protein sequences were retrieved from the National Center for Biotechnology Information (NCBI) [http://www.ncbi.nlm.nih.gov/]. The search of the protein database yielded 21 cyanobacterial sequences from which 10 cyanobacterial enzymes were selected after redundant sequences were excluded. Bacterial, mammalian, and plant sequences were also obtained from the NCBI protein database (Table 1). The sequences were converted to FASTA format using the ReadSeQ sequence conversion server [19].

2.2. Sequence Alignments. Multiple sequence alignments were performed with Clustal W [5]. The Clustal W alignment file was imported into the BoxShade sequence alignment editor. Identical and similar amino acids were shaded or colored. Phylogenetic analysis of protein sequences was generated using the alignment obtained with Clustal W.

2.3. Structural Analysis. The amino acid composition of the cyanobacterial PEPCK sequences was computed using the PEPSTATS analysis tool [24]. The physicochemical parameters such as the molecular weight, isoelectric point (pI), extinction coefficient, half-life, aliphatic index, amino acid property, instability index, and grand average hydropathy (GRAVY) were calculated using the ProtParam tool of the ExPASy proteomics server. Secondary structure elements prediction was performed using the Network Protein Sequence Analysis (NPS@) server and the Secondary Structural Content Prediction (SSCP) server [6, 25]. The consensus secondary structure content and predicted disulfide patterns of each cyanobacterial PEPCK are tabulated in Table 3. The presence of disulfide bridges was analyzed using the CYS-REC tool which predicts the most probable bonding patterns between available cysteine residues (http://linuxl.softberry.com/berry.phtml). The 3D models of cyanobacterial PEPCKs were constructed using the protein structure homology model building program SWISSMODEL with energy minimization parameters [21]. The modeled tertiary structures were built on the basis of sequence identity with the high-resolution crystal structures of the enzyme from Thermus thermophilus. The Swiss PDB viewer [26] was used to visualize and refine the models, and PyMOL (Schrödinger Inc.) was used to generate publishable images of the PEPCK models. The modeled 3D structures were evaluated and validated with the WHAT IF and RAMPAGE programs $[22,27]$.

2.4. Molecular Docking. The molecular operating environment (MOE) program was used to calculate the docking energies between oxaloacetate (OAA) and the active site of Cyanothece sp. PCC 7424 PEPCK. OAA was drawn using the MOE software [23]. The PDB file of the modeled Cyanothece sp. PCC 7424 PEPCK was imported into MOE, and a binding pocket in the area between the $\mathrm{C}$-terminal and $\mathrm{N}$-terminal domains was selected as the target area in a $3 \mathrm{D}$ docking box. OAA was imported to the protein's 3D box, and the docking program was initiated to search for favourable binding configurations between OAA and Cyanothece sp. PCC 7424 PEPCK. MOE's docking program flexes the substrate and attempts to fit it into the active site using an energy minimization algorithm. Docking studies were carried out at the Laboratory of Molecular Computations and Bioinformatics at Howard University, Washington, DC.

\section{Results and Discussion}

The primary structure analysis of cyanobacterial phosphoenolpyruvate carboxykinase enzymes showed that the most abundant amino acid is leucine which accounts for $9 \%$ of the enzyme's primary structure. The least common amino acids were tryptophan and cysteine. Cysteine residues are much more prevalent in GTP-dependent PEPCKs as such a greater number of disulfide bridges were predicted for 
TABLE 2: Parameters of cyanobacterial PEPCs calculated using the ProtParam program: molecular weight (MW) (g/mol); isoelectric point (pI); extinction coefficient (EC) $\left(\mathrm{M}^{-1} \mathrm{~cm}^{-1}\right)$; instability index (Ii); aliphatic index (Ai); grand average hydropathy (GRAVY); number of negative residues $(-\mathrm{R})$; number of positive residues $(+\mathrm{R})[6]$.

\begin{tabular}{|c|c|c|c|c|c|c|c|c|c|}
\hline Organism & Seq length & MW & $\mathrm{pI}$ & EC & Ii & $\mathrm{Ai}$ & GRAVY & $-\mathrm{R}$ & $+\mathrm{R}$ \\
\hline Cyanothece sp. PCC 8802 & 571 & 63940.3 & 6.07 & 72770 & 33.35 & 84.36 & -0.309 & 63 & 50 \\
\hline Cyanothece sp. PCC 7424 & 572 & 63646.3 & 6.06 & 58790 & 34.49 & 85.59 & -0.228 & 64 & 53 \\
\hline Cyanothece sp. PCC 8801 & 571 & 63966.3 & 6.07 & 72770 & 33.83 & 85.04 & -0.299 & 63 & 50 \\
\hline Cyanothece sp. ATCC 51142 & 569 & 63388.7 & 5.96 & 60280 & 28.65 & 84.01 & -0.262 & 62 & 50 \\
\hline Microcystis aeruginosa NIES-843 & 540 & 60169.2 & 5.60 & 65780 & 29.75 & 85.07 & -0.226 & 63 & 47 \\
\hline Cyanothece sp. CCY0110 & 568 & 63649.1 & 6.09 & 60780 & 33.59 & 84.98 & -0.283 & 62 & 51 \\
\hline Cyanothece sp. PCC 7822 & 612 & 67961.3 & 6.42 & 58790 & 37.70 & 84.30 & -0.260 & 65 & 58 \\
\hline Microcoleus chthonoplastes PCC 7420 & 555 & 61947.4 & 5.82 & 61770 & 32.67 & 83.44 & -0.268 & 63 & 51 \\
\hline Microcystis aeruginosa PCC 7806 & 540 & 60123.2 & 5.53 & 65780 & 30.22 & 85.80 & -0.223 & 64 & 47 \\
\hline Arthrospira platensis str. Paraca & 573 & 63546.8 & 5.45 & 67270 & 33.70 & 87.09 & -0.240 & 73 & 56 \\
\hline Trypanosoma cruzi & 472 & 52585.2 & 8.20 & 55350 & 41.65 & 77.50 & -0.254 & 56 & 59 \\
\hline E. coli $\mathrm{K} 12$ & 540 & 59643.4 & 5.46 & 74830 & 21.19 & 78.24 & -0.315 & 68 & 57 \\
\hline A succiniciproducens & 532 & 58642.8 & 5.77 & 68870 & 21.81 & 79.96 & -0.233 & 67 & 61 \\
\hline C. glutamicum & 610 & 66874.3 & 4.64 & 128340 & 36.86 & 75.74 & -0.295 & 89 & 51 \\
\hline Homo sapiens & 622 & 69177.4 & 5.80 & 104390 & 44.95 & 80.48 & -0.282 & 77 & 66 \\
\hline Rattus norvegicus & 622 & 69415.7 & 6.09 & 108860 & 44.43 & 79.53 & -0.308 & 78 & 71 \\
\hline Gallus gallus & 640 & 70970.1 & 8.16 & 122380 & 53.61 & 72.09 & -0.329 & 71 & 74 \\
\hline Thermus thermophilus HB8 & 529 & 59314.4 & 6.27 & 78270 & 39.59 & 79.96 & -0.282 & 65 & 60 \\
\hline
\end{tabular}

TABLE 3: Predicted consensus secondary structure content and predicted disulfide patterns of cyanobacterial PEPCs. The data was generated from the Protein Sequence Analysis server and CYS REC (http://linuxl.softberry.com/berry.phtml).

\begin{tabular}{|c|c|c|c|c|}
\hline Organism & $\alpha$ - helix & $\beta$-sheet & Coil & Disulfide bridge prediction \\
\hline Cyanothece sp. PCC 8802 & 23.64 & 16.99 & 59.37 & $291-440$ \\
\hline Cyanothece sp. PCC 7424 & 26.05 & 16.26 & 57.69 & None \\
\hline Cyanothece sp. PCC 8801 & 23.64 & 17.51 & 58.84 & $291-440$ \\
\hline Cyanothece sp. ATCC 51142 & 23.55 & 20.21 & 56.24 & $288-437,310-370$ \\
\hline Microcystis aeruginosa NIES- 843 & 26.30 & 16.85 & 56.85 & None \\
\hline Cyanothece sp. CCY0110 & 23.77 & 18.66 & 57.57 & $287-369,370-432$ \\
\hline Cyanothece sp. PCC 7822 & 25.82 & 15.20 & 58.99 & None \\
\hline Microcoleus chthonoplastes PCC 7420 & 24.68 & 17.30 & 58.02 & None \\
\hline Microcystis aeruginosa PCC 7806 & 25.74 & 15.56 & 58.70 & None \\
\hline Arthrospira platensis str. Paraca & 23.56 & 17.98 & 58.46 & None \\
\hline Trypanosoma cruzi & 22.67 & 22.25 & 55.08 & $8-9$ \\
\hline E. coli $\mathrm{K} 12$ & 25.19 & 18.70 & 56.11 & None \\
\hline A succiniciproducens & 25.56 & 18.23 & 56.20 & None \\
\hline C. glutamicum & 31.31 & 15.57 & 53.11 & $90-199$ \\
\hline Homo sapiens & 26.21 & 16.24 & 57.56 & $192-245,212-413$, and $399-407$ \\
\hline Rattus norvegicus & 27.17 & 18.01 & 54.82 & $192-399,245-307$, and $407-413$ \\
\hline Gallus gallus & 27.50 & 17.81 & 54.69 & $11-93,325-425$, and $417-431$ \\
\hline Thermus thermophilus HB8 & 25.90 & 19.47 & 54.63 & None \\
\hline
\end{tabular}

GTP-dependent enzymes (Table 3). The computed isoelectric point (pI) of cyanobacterial PEPCKs was 6.03 on average; this indicates that the enzyme is likely to precipitate in acidic buffers (Table 2). The extinction coefficient (EC) of cyanobacterial and prokaryotic PEPCKs was lower than that of GTP-dependent enzymes. The instability indices (Ii) of bacterial PEPCKs were below 40, indicating that they would be stable in solution. The ProtParam tool calculated instability indices above 40 for selected eukaryotic PEPCKs [6]. All PEPCKs have negative GRAVY scores attesting to their solubility in hydrophilic solvents. The aliphatic index (Ai) which evaluates the relative volume occupied by the side chains of hydrophobic amino acids was generally higher in bacterial PEPCKs. A high aliphatic index indicates that a protein may remain stable over a wide range of temperatures. 


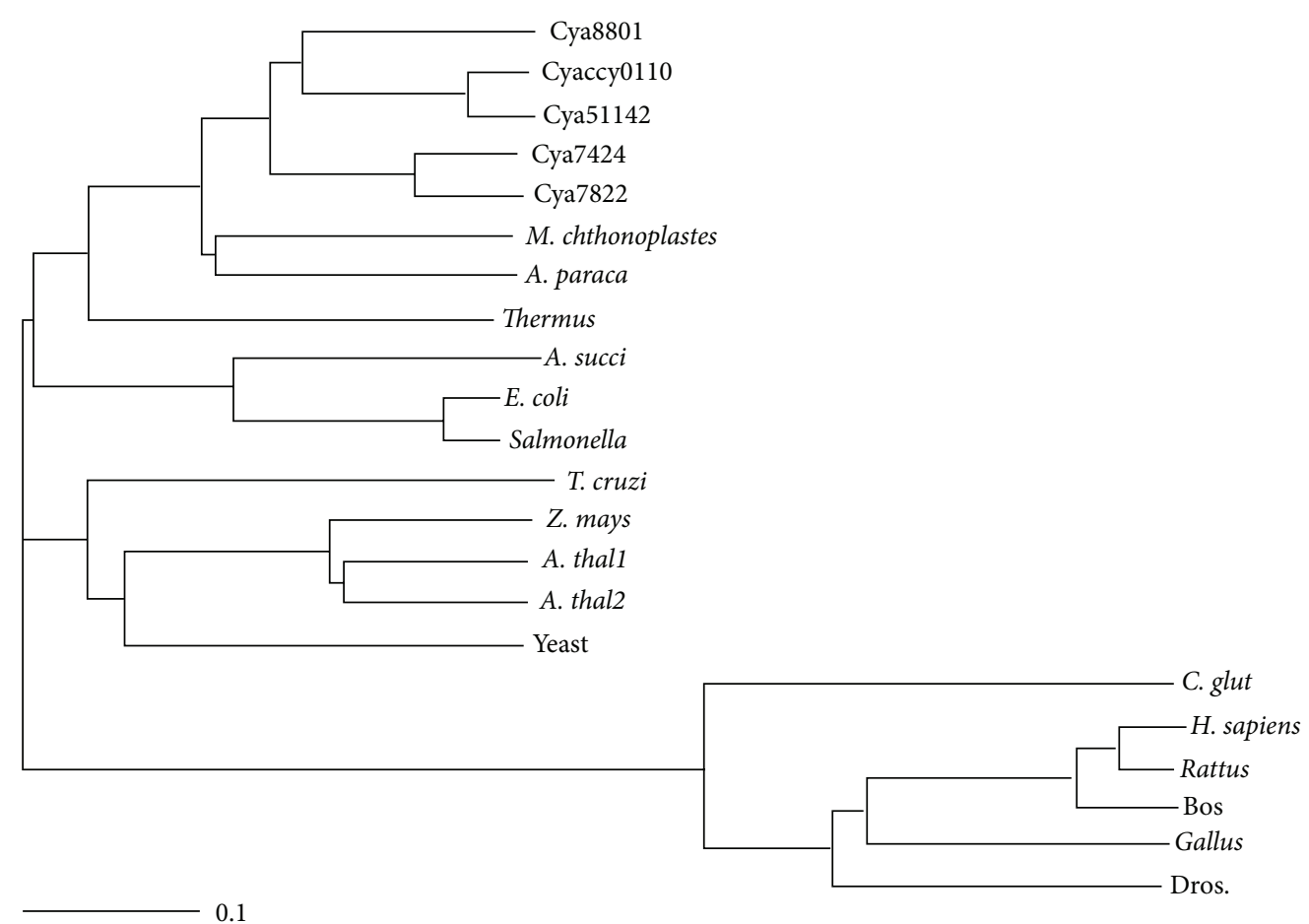

FIGURE 2: Cladogram of selected PEPCKs based on amino acid sequences of ATP- and GTP-dependent enzymes.

A multiple sequence alignment of selected ATP- and GTP-dependent PEPCKs was performed (Figure 1). While sequence identity was insignificant between the two types, domains involved in catalysis and metal ion coordination were conserved between ATP- and GTP-dependent PEPCKs. A phylogenetic tree was constructed from the alignment (Figure 2). The cladogram illustrated the high degree of divergence between ATP- and GTP-utilizing enzymes. The multiple sequence alignment also revealed amino acid sequences that are only found in cyanobacterial enzymes and do not occur in PEPCKs from eukaryotes and other prokaryotic organisms (Figure 1).

The predicted secondary structure composition of Cyanothece sp. PCC 7424 PEPCK was determined using the NPS@ server which generates a consensus report from twelve secondary structure prediction methods [6]. All PEPCKs shared similar alpha helical and $\beta$-sheet content (Table 3). A more detailed analysis of the secondary structural elements of Cyanothece sp. PCC 7424 PEPCK was performed using the PDBsum tool [20]. The secondary structure prediction server revealed that $26.05 \%$ of amino acids resided in $\alpha$ helices, while $16.26 \%$ of residues were in $\beta$-sheets. The rest of the amino acids were found in other conformations such as $\beta$-hairpins and $\beta$-turns. The topology of Cyanothece sp. PCC 7424 PEPCK is illustrated in Figure 3(b). The overall fold of the enzyme is similar to other PEPCKs for which crystal structures are available. The protein's main chain consists of a $\mathrm{C}$-terminal domain and an N-terminal domain that are both folded into a mixed $\alpha / \beta$ topology.

The predicted secondary structures generated by the PDBsum tool were generally in agreement with the three-dimensional structure of Cyanothece sp. PCC 7424 PEPCK (Figure 3(a)). However, while the enzyme's computed schematic diagram illustrated the existence of $\alpha$-helices and $\beta$-sheets in the $\mathrm{N}$-terminal between residues 50 and 88 , no such structures exist in the corresponding region of the 3D model. This may be due to the fact that PEPCKs across species align poorly in this particular area of the $\mathrm{N}$-terminal domain. According to the schematic diagram, the active site lid ${ }^{417}$ SKLAGTERGITAP ${ }^{428}$ is located between two $\beta$ strands. This was accurately represented in the $3 \mathrm{D}$ structure. The same can be said for the locations of the kinase-la and kinase 2 motifs as well as the ATP-binding site.

The homology model of Cyanothece sp. PCC 7424 PEPCK was generated with the SWISS-MODEL software using Thermus thermophilus PEPCK as a template (Figure 4). The overall fold of the Cyanothece sp. PCC 7424 enzyme is similar to that of Thermus thermophilus PEPCK as expected from a sequence identity score of $51 \%$ between the two proteins. According to the Ramachandran plot generated with the RAMPAGE server, $96.2 \%$ of residues are found in the most favoured region, while $2.7 \%$ of amino acids reside in the generously allowed region (Figure 5). Furthermore, the model's calculated QMEAN4 score of 0.771 long with a Z score 0.3 confirmed its reliability [28]. The protein is organized into a C-terminal domain and an N-terminal domain with mixed $\alpha / \beta$ content. The active site is located in a deep cleft between the two domains.

Based on what is known of ATP-dependent PEPCKs the purported active site residues of Cyanothece sp. PCC 7424 PEPCK are R100, K240, K241, H260, K282, D297, K316, and R362. In the well-characterized E. coli enzyme, the lysine 


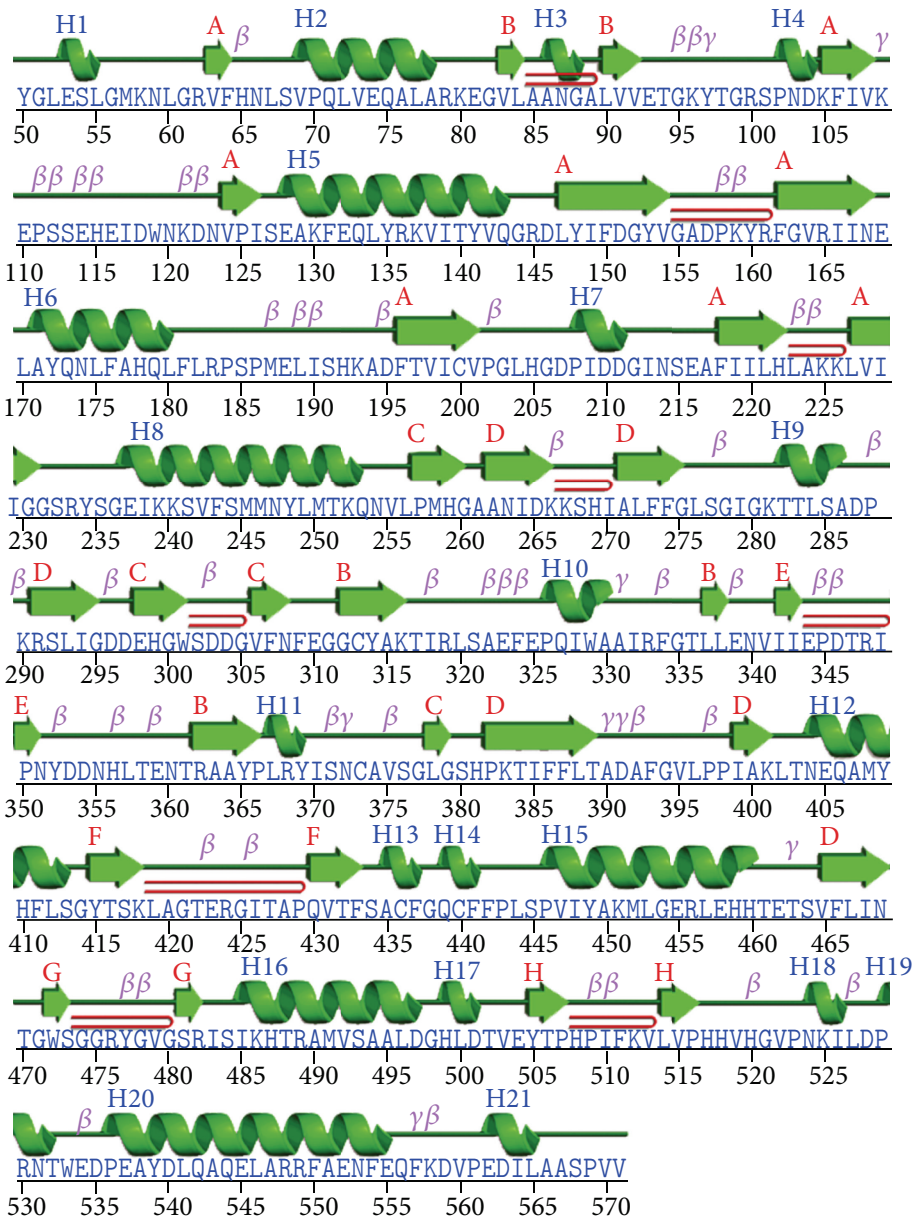

(a)

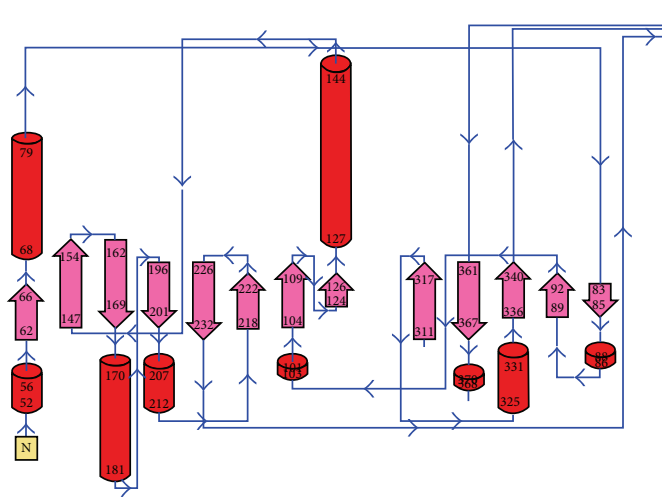

$\mathrm{N}$-terminal domain

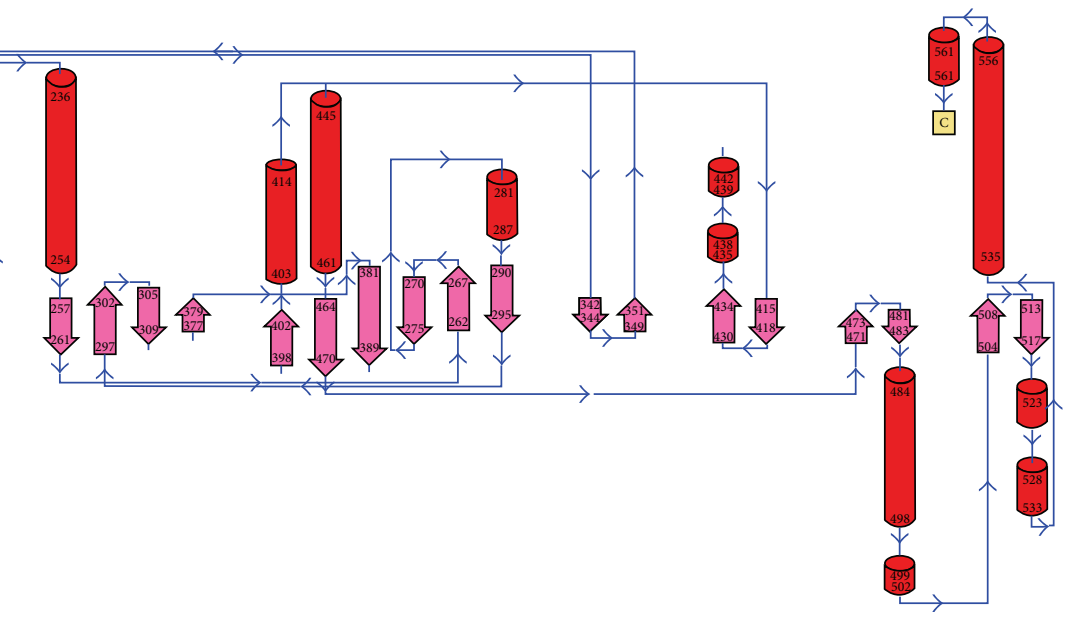

C-terminal domain

(b)

FIGURE 3: Schematic and topology diagrams showing the secondary structural elements in the Cyanothece sp. PCC 7424 PEPCK. (a) $\alpha$-helices are labeled with the letter " $\mathrm{H}$ ", and $\beta$-strands are lettered in uppercase. $\beta, \gamma$, and hairpin turns are also labeled. (b) Helices are represented as cylinders and $\beta$-strands as arrows. The secondary motif map and topology diagram were calculated using the PDBsum tool [20]. 


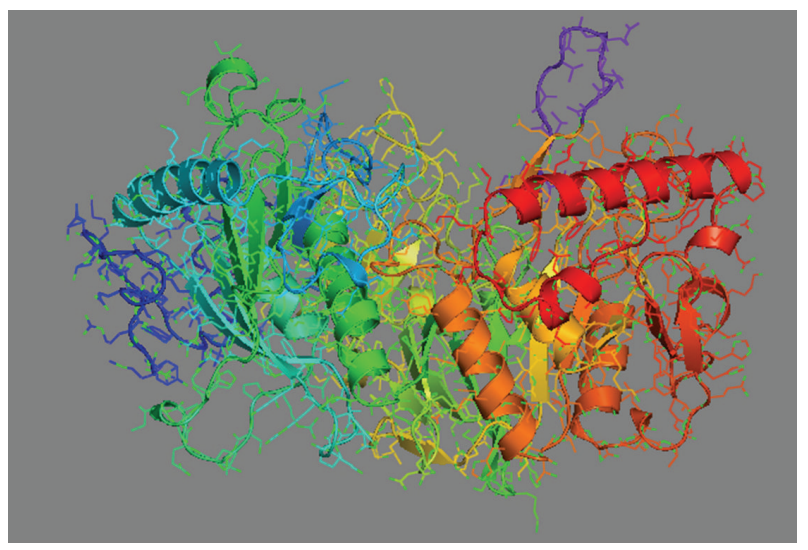

FIGURE 4: Predicted 3D structure of the Cyanothece sp. PCC 7424 PEPCK. The models were generated with SWISS-MODEL using PDB template 1xkvB. PyMOL was used to visualize the model. The active side lid is shown as a purple loop in the C-terminal domain [21].
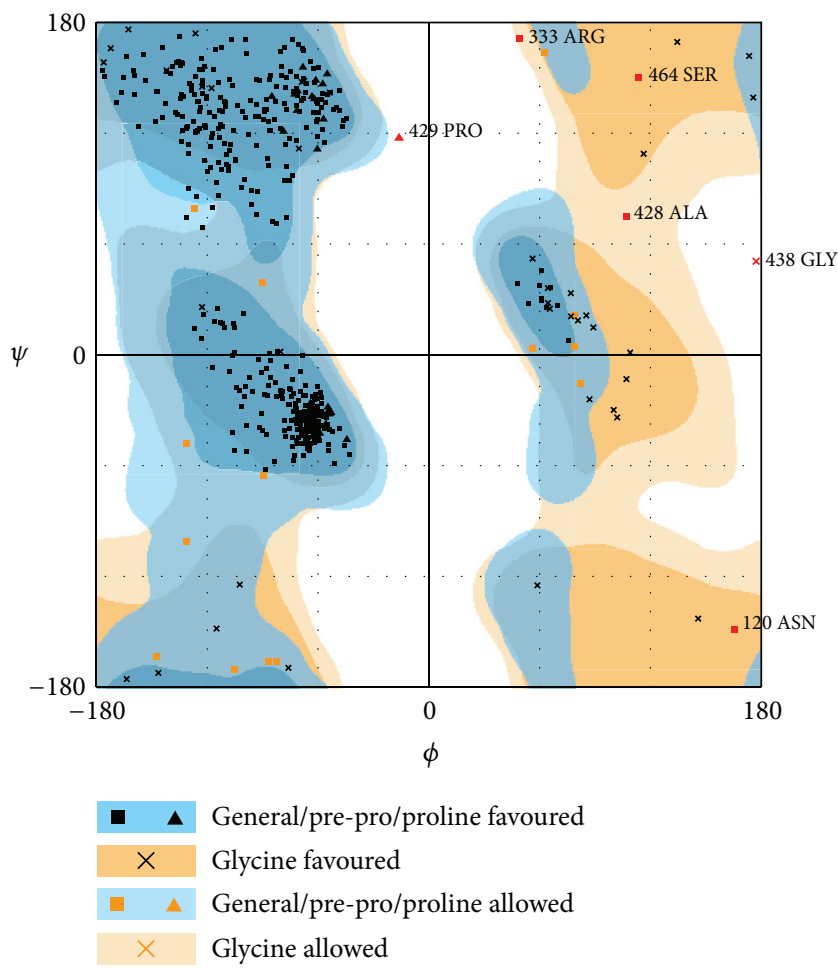

FIgURE 5: The Ramachandran plot for the modeled Cyanothece sp. PCC 7424 PEPCK. 96.2\% of residues are found in the most favoured regions, $2.7 \%$ of residues are in the most allowed regions, and $1.1 \%$ of residues are found I the outlier regions. The plot was generated with the RAMPAGE program [22].

residues homologous to K240 and K241 are involved in OAA and PEP binding [28]. Furthermore, R100 along with R362 are thought to be involved in pyruvate binding; additionally, R100 and $\mathrm{Y} 235$ form $\mathrm{H}$-bonds with $\mathrm{CO}_{2}$ [29]. The residues form a positively charged active site that is well suited for OAA and PEP binding (Figure 6). Metal ion coordination is also

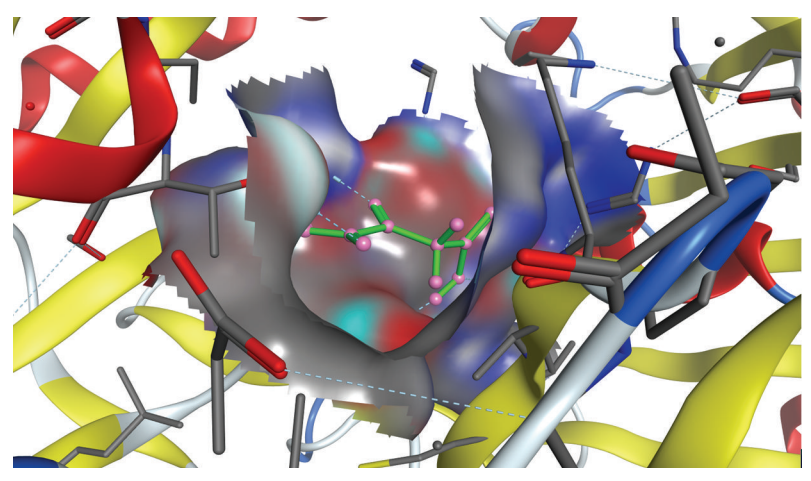

FIgURE 6: The electrostatic surface representation of the active site of Cyanothece sp. PCC 7424 PEPCK generated by MOE [23]. The surface residues are color coded based on their nature: the blue coloration indicates that the enzyme's substrate-binding pocket is positively charged (MOE; Chemical Computing Group, Inc.).

an important aspect of the catalytic mechanism of PEPCK. In Cyanothece sp. PCC 7424 PEPCK, active site residues H260, D297, and K316 are most likely involved in $\mathrm{Mn}^{2+}$ or $\mathrm{Mg}^{2+}$ binding. Furthermore the cyanobacterial protein features the sequence ${ }^{306}$ IFNFEGCYAK ${ }^{315}$ which forms the $\mathrm{Ca}^{2+}$-binding domain in ATP-dependent PEPCKs [30].

Cyanobacterial PEPCKs also feature the nucleotidebinding motif kinase-la which consensus sequence is GXXGXGKT [31]. This motif, which forms the phosphatebinding loop, is common in ATPase enzymes. Another motif found in cyanobacterial PEPCKs is kinase 2 with the consensus sequence $\mathrm{XXXXD}$, where $\mathrm{X}$ is a hydrophobic amino acid. This motif is often present in nucleotide-binding proteins. In the C-terminal domain of Cyanothece sp. PCC 7424 PEPCK, the kinase-la motif is ${ }^{276} \mathrm{GLSGTGKT}^{283}$, and the kinase 2 motif is ${ }^{293}$ LIGDD $^{297}$. In E. coli PEPCK, D296 and D297 are involved in $\mathrm{Mn}^{2+}$ binding. The N-terminal domain of Cyanothece sp. PCC 7424 PEPCK includes two additional motifs that appear to be exclusive to cyanobacterial enzymes: ${ }^{48}$ PTYGLE $^{53}$ and ${ }^{203}$ GLHGDPE/ ${ }^{209}$. The adenine-binding motif, ${ }^{482}$ RIPIKHT ${ }^{488}$, is found in the C-terminal of Cyanothece sp. PCC 7424 PEPCK. H487 is found in all cyanobacterial sequences, while the equivalent amino acid is found as either a valine or aspartate residue in other ATP-dependent PEPCKs (Figure 1).

The sequence ${ }^{417}$ SKLAGTERGITAP ${ }^{428}$ makes up the active site lid which is prevalent in ATP-utilizing PEPCKs [32]. This loop stands above the active site cleft and prevents water molecules from interring with the substrate (Figure 4). The $\Omega$-loop is also present in GTP-dependent enzymes, but it has a different location and sequence. The $3 \mathrm{D}$ model of Cyanothece sp. PCC 7424 PEPCK generated with the SWISS-MODEL software was that of the unliganded, lid open conformation of the enzyme. Additional models of the protein were built with the Phyre2 and ESyPred3D automated homology modeling programs using default parameters [33, 34]. The generated $3 \mathrm{D}$ structures depicted the enzyme in the 


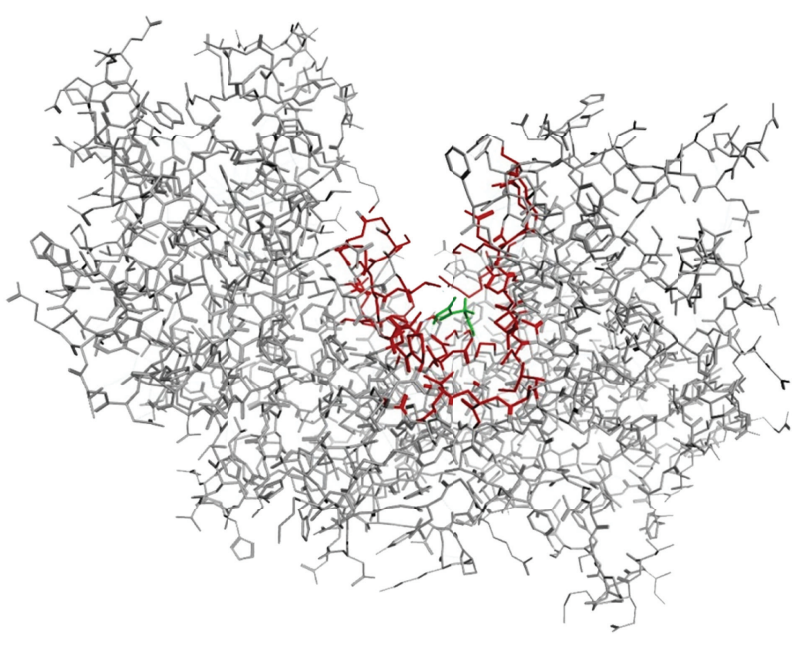

(a)

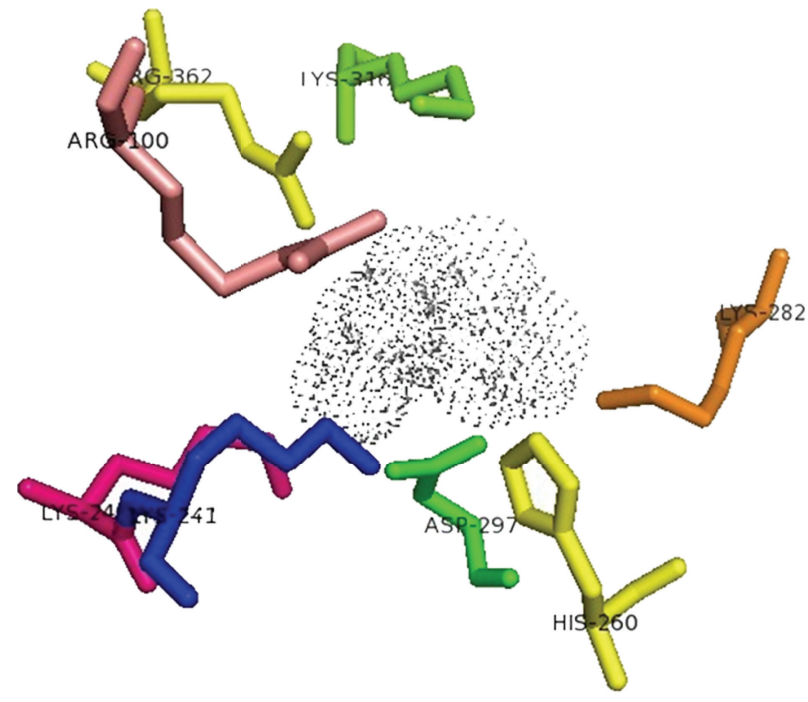

(b)

FIgURE 7: (a) Predicted 3D structure of liganded Cyanothece sp. PCC 7424 PEPCK. The binding pocket is shown in red with the bound OAA substrate in green. (b) Active site of Cyanothece sp. PCC 7424 PEPCK showing active site residues as sticks and OAA as spheres. The model was generated with SWISS-MODEL, and docking studies were performed with MOE (Chemical Computing Group, Inc.); [21, 23].

lid closed conformation as both programs used the ATP$\mathrm{Mg}^{2+}$-oxalate ternary complex of E. coli PEPCK [35].

The MOE software correctly predicted the binding site of Cyanothece sp. PCC 7424 PEPCK (Figure 7). While the program flexes the substrate, the conformation of the modeled protein is left unchanged when energy minimization calculations are being performed. The substrate is able to bind to the active site when the lid is in the open conformation, but the lid must close in order for all active site residues to interact with the substrate and to initiate catalysis [36]. Furthermore, the binding of ATP to the nucleotide binding site causes a conformational change which correlates to lid closure and catalytic activity [37].

\section{Conclusion}

This study provided the 3D structure of Cyanothece sp. PCC 7424 PEPCK through a homology modeling approach. This particular enzyme was modeled because of this group's particular interest in investigating the role of PEPCK in overall carbon assimilation by Cyanothece sp. PCC 7424 an obligate autotroph with unique metabolic properties. Docking studies of the interaction of OAA with the enzyme's active site showed that the spatial arrangement of catalytic amino acids in the cyanobacterial enzyme was similar to that of other modeled PEPCKs; this suggests a catalytic mechanism that is similar to other forms of the enzyme. In general, the enzyme's primary structure varies considerably among species suggesting significant evolutionary divergence. The precise role of PEPCK in cyanobacteria has yet to be investigated, and whether PEPCK is present in marine cyanobacteria remains to be seen. More work will be needed to determine the potential role of PEPCK in cyanobacterial carbon fixation.

\section{Acknowledgment}

The authors are grateful to Dr. Yayin Fang of the Center for Computational Biology and Bioinformatics at Howard University for the use of computational facilities and her invaluable expertise during the docking studies.

\section{References}

[1] G. M. Carlson and T. Holyoak, "Structural insights into the mechanism of phosphoenolpyruvate carboxykinase catalysis," Journal of Biological Chemistry, vol. 284, no. 40, pp. 2703727041, 2009.

[2] P. Dunten, C. Belunis, R. Crowther et al., "Crystal structure of human cytosolic phosphoenolpyruvate carboxykinase reveals a new GTP-binding site," Journal of Molecular Biology, vol. 316, no. 2, pp. 257-264, 2002.

[3] S. Aich, L. Prasad, and L. T. J. Delbaere, "Structure of a GTPdependent Bacterial PEP-carboxykinase from Corynebacterium glutamicum," International Journal of Biochemistry and Cell Biology, vol. 40, no. 8, pp. 1597-1603, 2008.

[4] Y. A. Leduc, L. Prasad, M. Laivenieks, J. G. Zeikus, and L. T. J. Delbaere, "Structure of PEP carboxykinase from the succinateproducing Actinobacillus succinogenes: a new conserved activesite motif," Acta Crystallographica Section D, vol. 61, no. 7, pp. 903-912, 2005.

[5] M. A. Larkin, G. Blackshields, N. P. Brown et al., "Clustal W and Clustal X version 2.0," Bioinformatics, vol. 23, no. 21, pp. 29472948, 2007.

[6] E. Gasteiger, C. Hoogland, A. Gattiker et al., "Protein identification and analysis tools on the ExPASy server," in The Proteomics Protocols Handbook, J. M. Walker, Ed., pp. 571-607, Humana Press, 2005. 
[7] J. Yang, S. C. Kalhan, and R. W. Hanson, "What is the metabolic role of phosphoenolpyruvate carboxykinase?" Journal of Biological Chemistry, vol. 284, no. 40, pp. 27025-27029, 2009.

[8] N. Asanuma, K. Kanada, Y. Arai, K. Yoshizawa, T. Ichikawa, and T. Hino, "Molecular characterization and significance of phosphoenolpyruvate carboxykinase in a ruminal bacterium, streptococcus bovis," Journal of General and Applied Microbiology, vol. 56, no. 2, pp. 121-127, 2010.

[9] Y. D. Kwon, S. Y. Lee, and P. Kim, "A physiology study of Escherichia coli overexpressing phosphoenolpyruvate carboxykinase," Bioscience, Biotechnology and Biochemistry, vol. 72, no. 4, pp. 1138-1141, 2008.

[10] R. M. Zelle, J. Trueheart, J. C. Harrison, J. T. Pronk, and A. J. A. Van Maris, "Phosphoenolpyruvate carboxykinase as the sole anaplerotic enzyme in saccharomyces cerevisiae," Applied and Environmental Microbiology, vol. 76, no. 16, pp. 5383-5389, 2010.

[11] R. P. Walker, Z. H. Chen, R. M. Acheson, and R. C. Leegood, "Effects of phosphorylation on phosphoenolpyruvate carboxykinase from the $\mathrm{C}_{4}$ plant Guinea grass," Plant Physiology, vol. 128, no. 1, pp. 165-172, 2002.

[12] M. Martín, S. P. Rius, and F. E. Podestá, “Two phosphoenolpyruvate carboxykinases coexist in the Crassulacean Acid Metabolism plant Ananas comosus. Isolation and characterization of the smaller $65 \mathrm{kDa}$ form," Plant Physiology and Biochemistry, vol. 49, no. 6, pp. 646-653, 2011.

[13] J. R. Reinfelder, A. J. Milligan, and F. M. M. Morel, “The role of the $\mathrm{C}_{4}$ pathway in carbon accumulation and fixation in a marine diatom," Plant Physiology, vol. 135, no. 4, pp. 2106-2111, 2004.

[14] J. Xu, X. Fan, X. Zhang et al., "Evidence of coexistence of $C_{3}$ and $\mathrm{C}_{4}$ photosynthetic pathways in a green-tide-forming alga, Ulva prolifera," PLoS ONE, vol. 7, no. 5, Article ID e37438, 2012.

[15] A. A. Smith, M. W. Coomes, and T. E. Smith, "Isolation and sequence of the phosphoenolpyruvate carboxylase gene of the marine cyanobacterium Synechococcus PCC 7002," Journal of Biological Sciences, vol. 8, no. 8, pp. 1261-1270, 2008.

[16] B. O'Leary, S. K. Rao, J. Kim, and W. C. Plaxton, "Bacterialtype phosphoenolpyruvate carboxylase (PEPC) functions as a catalytic and regulatory subunit of the novel class-2 PEPC complex of vascular plants," Journal of Biological Chemistry, vol. 284, no. 37, pp. 24797-24805, 2009.

[17] S. R. Smith, R. M. Abbriano, and M. Hildebrand, "Comparative analysis of diatom genomes reveals substantial differences in the organization of carbon partitioning pathways," Algal Research, vol. 1, no. 1, pp. 2-16, 2012.

[18] A. A. Smith and M. C. Plazas, "In silico characterization and homology modeling of cyanobacterial Phosphoenolpyruvate carboxylase enzymes with computational tools and bioinformatics servers," American Journal of Biochemistry and Molecular Biology, vol. 1, no. 4, pp. 319-336, 2011.

[19] D. Gilbert, "Sequence file format conversion with commandline readseq," in Current Protocols in Bioinformatics, A. D. Baxevanis, G. A. Petsko, L. D. Stein, and G. D. Stormo, Eds., John Wiley \& Sons, Hoboken, NJ, USA, 2003.

[20] R. A. Laskowski, V. V. Chistyakov, and J. M. Thornton, "PDBsum more: new summaries and analyses of the known 3D structures of proteins and nucleic acids," Nucleic Acids Research, vol. 33, pp. D266-D268, 2005.

[21] K. Arnold, L. Bordoli, J. Kopp, and T. Schwede, "The SWISSMODEL workspace: a web-based environment for protein structure homology modelling," Bioinformatics, vol. 22, no. 2, pp. 195-201, 2006.
[22] S. C. Lovell, I. W. Davis, W. B. Arendall et al., "Structure validation by $\mathrm{C} \alpha$ geometry: $\varphi, \psi$ and $\mathrm{C} \beta$ deviation," Proteins: Structure, Function and Genetics, vol. 50, no. 3, pp. 437-450, 2003.

[23] Molecular Operating Environment (MOE), 2012.10, Chemical Computing Group, Montreal, Quebec, Canada, 2012.

[24] P. Rice, L. Longden, and A. Bleasby, "EMBOSS: the European molecular biology open software suite," Trends in Genetics, vol. 16, no. 6, pp. 276-277, 2000.

[25] F. Eisenhaber, F. Imperiale, P. Argos, and C. Frommel, "Prediction of secondary structural content of proteins from their amino acid composition alone. I. New analytic vector decomposition methods," Proteins, vol. 25, no. 2, pp. 157-168, 1996.

[26] N. Guex and M. C. Peitsch, "SWISS-MODEL and the SwissPdbViewer: an environment for comparative protein modeling," Electrophoresis, vol. 18, no. 15, pp. 2714-2723, 1997.

[27] G. Vriend, "WHAT IF: a molecular modeling and drug design program," Journal of Molecular Graphics, vol. 8, no. 1, pp. 52-56, 1990.

[28] P. Benkert, M. Biasini, and T. Schwede, "Toward the estimation of the absolute quality of individual protein structure models," Bioinformatics, vol. 27, no. 3, pp. 343-350, 2011.

[29] C. Andrade, C. Sepulveda, E. Cardemil, and A. M. Jabalquinto, "The role of tyrosine 207 in the reaction catalyzed by Saccharomyces cerevisiae phosphoenolpyruvate carboxykinase," Biological Research, vol. 43, no. 2, pp. 191-195, 2010.

[30] M. Sugahara, N. Ohshima, Y. Ukita, M. Sugahara, and N. Kunishima, "Structure of ATP-dependent phosphoenolpyruvate carboxykinase from Thermus thermophilus HB8 showing the structural basis of induced fit and thermostability," Acta Crystallographica Section D, vol. 61, no. 11, pp. 1500-1507, 2005.

[31] S. Trapani, J. Linss, S. Goldenberg, H. Fischer, A. F. Craievich, and G. Oliva, "Crystal structure of the dimeric phosphoenolpyruvate carboxykinase (PEPCK) from Trypanosoma cruzi at $2 \AA$ resolution," Journal of Molecular Biology, vol. 313, no. 5, pp. 1059-1072, 2001.

[32] J. J. H. Cotelesage, L. Prasad, J. G. Zeikus, M. Laivenieks, and L. T. J. Delbaere, "Crystal structure of Anaerobiospirillum succiniciproducens PEP carboxykinase reveals an important active site loop," International Journal of Biochemistry and Cell Biology, vol. 37, no. 9, pp. 1829-1837, 2005.

[33] L. A. Kelley and M. J. Sternberg, "Protein structure prediction on the web: a case study using the Phyre server," Nature Protocols, vol. 4, no. 3, pp. 363-371, 2009.

[34] C. Lambert, N. Léonard, X. De Bolle, and E. Depiereux, "ESyPred3D: prediction of proteins 3D structures," Bioinformatics, vol. 18, no. 9, pp. 1250-1256, 2002.

[35] L. W. Tari, A. Matte, U. Pugazhenthi, H. Goldie, and L. T. J. Delbaere, "Snapshot of an enzyme reaction intermediate in the structure of the ATP- $\mathrm{Mg}^{2+}$-oxalate ternary complex of Escherichia coli PEP carboxykinase," Nature Structural Biology, vol. 3, no. 4, pp. 355-363, 1996.

[36] S. M. Sullivan and T. Holyoak, "Enzymes with lid-gated active sites must operate by an induced fit mechanism instead of conformational selection," Proceedings of the National Academy of Sciences of the United States of America, vol. 105, no. 37, pp. 13829-13834, 2008.

[37] T. A. Johnson and T. Holyoak, "Increasing the conformational entropy of the $\Omega$-loop lid domain in phosphoenolpyruvate carboxykinase impairs catalysis and decreases catalytic fidelity," Biochemistry, vol. 49, no. 25, pp. 5176-5187, 2010. 

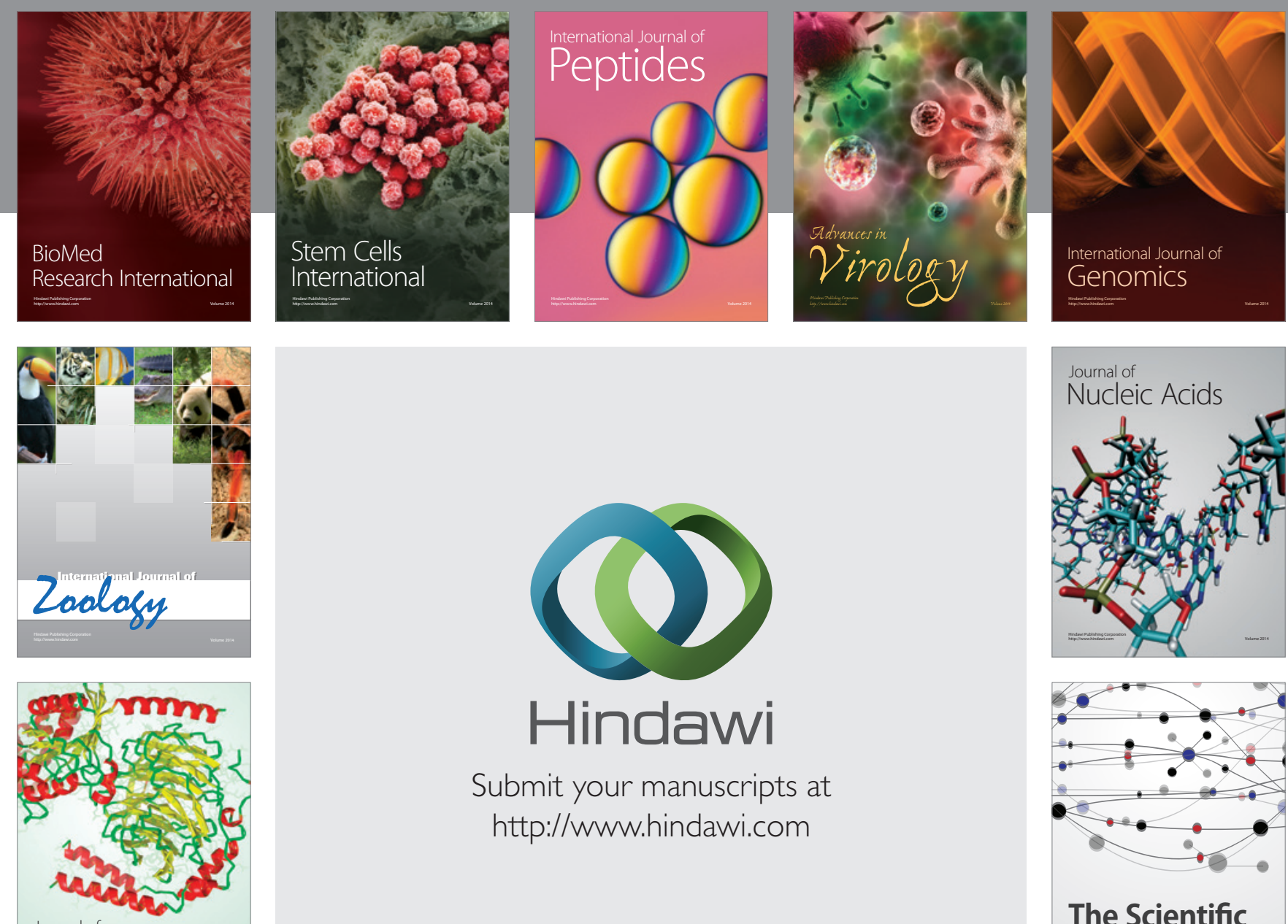

Submit your manuscripts at

http://www.hindawi.com

Journal of
Signal Transduction
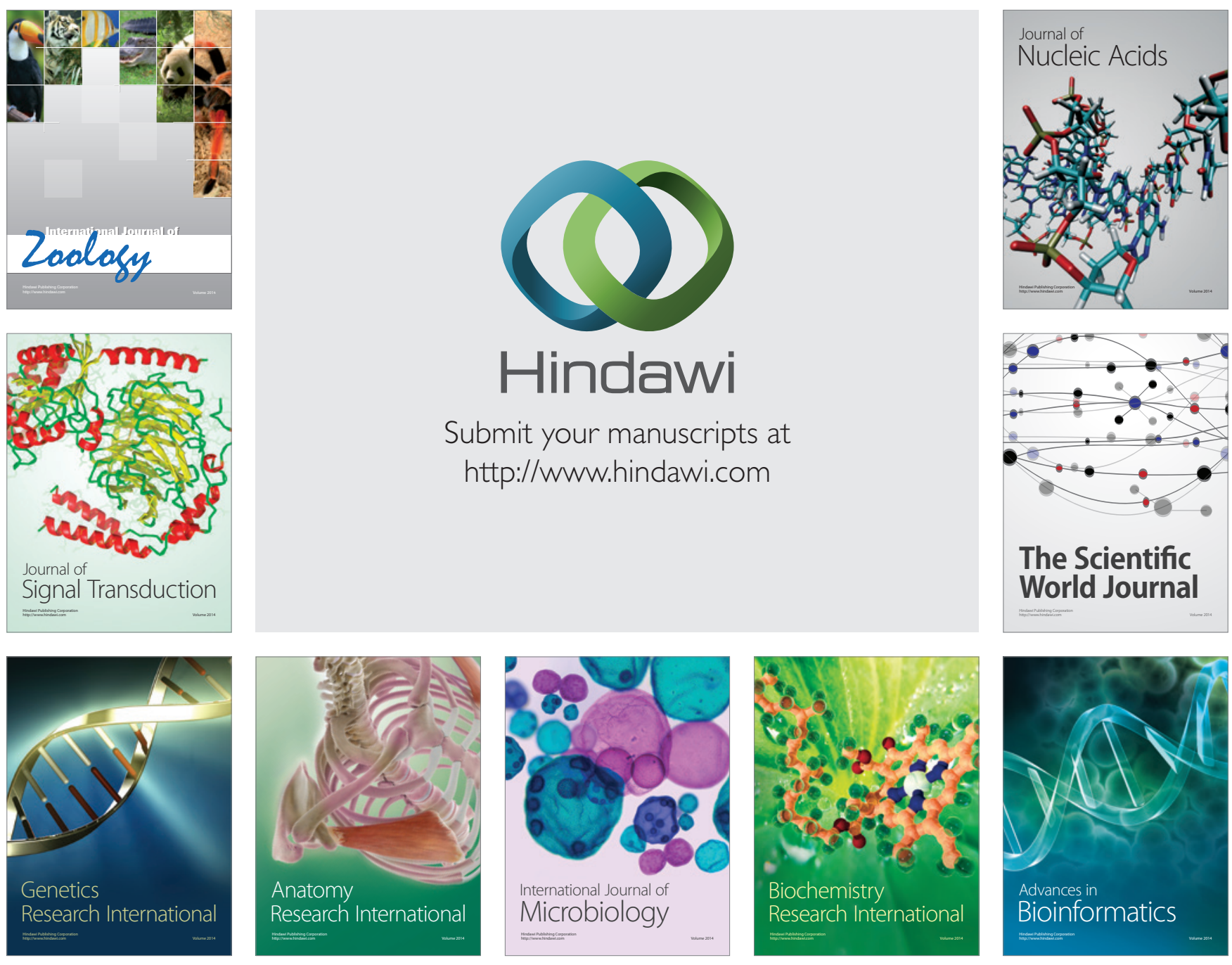

The Scientific World Journal
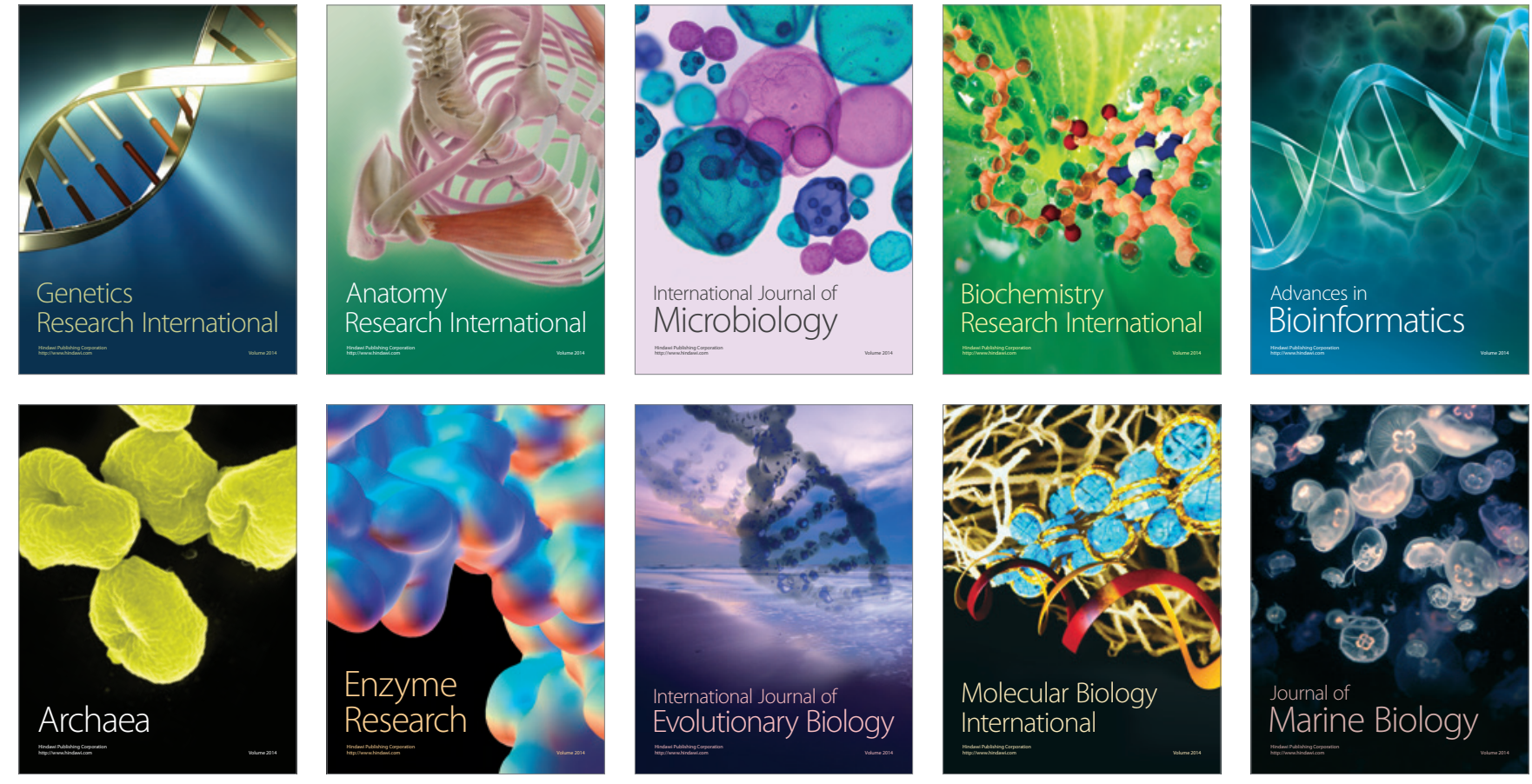